\title{
Schwerpunkte und Themen des Sammelbands
}

\author{
Christoph König und Katharina Mahrt
}

\section{1 Über das Publikationsprojekt}

Das Spektrum der Themen, die innerhalb der Hochschul-Community bearbeitet und vom Hochschulforum aufgegriffen wurden, hat sich seit 2014 kontinuierlich erweitert. Die Studien und Arbeits- und Diskussionspapiere, die das Hochschulforum publiziert, um über Entwicklungen und Trends zu informieren, spiegeln diese Themenvielfalt wider. ${ }^{1}$ 2016, zum Ende der ersten Projektphase, veröffentlichte das Hochschulforum einen umfassenden Sachstand unter dem Titel „The Digital Turn - Hochschulbildung im digitalen Zeitalter" (Hochschulforum Digitalisierung 2016). Bereits dieser Zwischenstand wurde von zahlreichen Expert*innen erarbeitet, die sich von 2014 bis 2016 in sechs Arbeitsgruppen statusgruppen- und länderübergreifend engagiert hatten.

Der vorliegende Sammelband schreibt diese Geschichte in einem neuen Format und unter dem programmatischen Titel ,Digitalisierung in Studium und Lehre gemeinsam gestalten“ fort. Das enorme Potenzial von kollaborativen Arbeitsprozessen und die Leistungsbereitschaft der HFD-Community zeigten sich in dem Zusammenhang einmal mehr: Fast 90 Vorschläge für Beiträge wurden innerhalb von zwei Monaten beim HFD eingereicht, von denen die Hälfte bis Mai 2020 ausgearbeitet wurden. Zudem erklärten sich 65 Wissenschaftler*innen bereit, als Gutachter*innen für den Sammelband zu fungieren. Für dieses einzigartige

\footnotetext{
${ }^{1}$ Alle HFD-Publikationen sind frei verfügbar unter: https://hochschulforumdigitalisierung. de/publikationen. Zugegriffen: 05.11.2020.

C. König $(\varangle) \cdot$ K. Mahrt

Stifterverband, Hochschulforum Digitalisierung, Berlin, Deutschland E-Mail: info@hochschulforumdigitalisierung.de
} 
Engagement bedankt sich das Hochschulforum Digitalisierung bei allen, die individuell und gemeinsam - Vorschläge und Beiträge erarbeitet, begutachtet und überarbeitet haben.

Dass am Ende dieses intensiven Prozesses nicht alle Vorschläge in den Band aufgenommen werden konnten, liegt in der Natur des neuen Publikationsformats. Das Hochschulforum Digitalisierung wagt sich mit dem vorliegenden Band erstmals an ein Medium, das den Kriterien der Scientific Community Rechnung trägt und damit auch die forschungsrelevanten Leistungen der HFDCommunity sichtbar macht. Mit Springer VS konnte das Hochschulforum einen starken Partner gewinnen, der die größtmögliche Verbreitung der Ergebnisse des Sammelbands im deutschsprachigen Hochschulraum verspricht und auch die Open-Access-Veröffentlichung unter der CC-BY-4.0-Lizenz ermöglicht hat.

Alle Beiträge wurden einem Double-Blind-Peer-Review-Verfahren unterzogen, um sicherzustellen, dass die Publikation die Standards guter wissenschaftlicher Praxis erfüllt. Die 33 Beiträge vermitteln im Zusammenspiel einen umfassenden Eindruck von den Herausforderungen und Chancen der digitalen Transformation für die Hochschulbildung. Große Potenziale liegen aus Sicht des Hochschulforums in kollaborativen und ganzheitlichen Ansätzen: Neue und häufig teure Technologien und Entwicklungen können zum Beispiel in hochschul- oder länderübergreifenden Kollaborationen für die Lehre gewonnen werden. Dies erfordert jedoch nicht nur eine entsprechende strategische Ausrichtung und ein Commitment von Hochschulleitungen, sondern gleichermaßen didaktische Innovationen von Lehrenden in - physischen oder virtuellen - Lernräumen.

Diesem Gedanken entsprechend adressiert der Sammelband ein breites Spektrum an Entwicklungen und Fragestellungen: innovative didaktische Formate und hybride Räume der Vermittlung von Wissen und (Zukunfts-)Kompetenzen, die Skalierung von Wissensvermittlung im Rahmen von Kooperationsprojekten oder Plattformen (lokal, regional, bundesweit oder international), die Inklusivität neuer Lehr-Lern-Settings und ihre soziale Dimension, Fragen der Integration neuer Lehrformate in bestehende Curricula sowie der Anerkennung von Lernleistungen (durch digitale Nachweise), datenschutzrechtliche und ethische Fragen im Zusammenhang mit der Nutzung digitaler Technologien und der Datafizierung des Studiums, bis hin zu Hochschulstrategien und politischen Rahmenbedingungen, die solche (kollaborativen) Versuche möglich machen. Die in diesem Band versammelten Beiträge werfen fundierte Schlaglichter auf die skizzierten Themen. Sie erfassen dabei nicht nur den Status quo, sondern zeigen viele mögliche Schritte zur Gestaltung der Digitalisierung in Studium und Lehre in den kommenden Jahren auf. 


\section{2 Überblick über die Beiträge des Sammelbands}

Der Band gliedert sich in fünf Teile, die im Folgenden kurz vorgestellt werden. Die Planung und Kuratierung des Sammelbandes erfolgte auch innerhalb des HFD-Teams in einem kollaborativen Prozess. So spiegeln die Beiträge in erster Linie die Themen wider, die aus Sicht des Hochschulforums und diverser Expert*innen aus der Community für die Gestaltung des digitalen Wandels entscheidend sein werden.

\subsection{Hochschulbildung und Digitalisierung}

Der erste Teil reflektiert breitere Entwicklungslinien und Trends. Dabei wird deutlich, dass die Gestaltung der Digitalisierung in erster Linie eine soziokulturelle Frage ist, die in den Hochschulen wie auch der hochschuldidaktischen und -politischen Community verhandelt wird.

Markus Deimann setzt sich kritisch mit dem Diskurs und dem Fortschrittsnarrativ der technologieunterstützten Hochschulbildung seit den 1990er-Jahren auseinander. Vor dem Hintergrund seiner Bestandsaufnahme der Fortschritte und Defizite im Bereich der digitalisierten Hochschullehre reflektiert Deimann die Erfahrungen der „Quick-and-Dirty-Digitalisierung“ im digitalen Sommersemester 2020 und plädiert für eine planvolle Politik der kleinen Schritte mit einer geteilten Vision.

Michael Jäckel betont die Resilienz der Hochschulen als Organisationsform des Wissens - auch und gerade in Zeiten von gesellschaftlichen Umbrüchen. Mit Blick auf die Digitalisierung folgert Jäckel, dass die damit verbundenen Herausforderungen nach den Regeln der Institution integriert würden. Viele Hochschulen und Universitäten erwiesen sich aber bereits heute als weitaus digitaler, als sie in der Öffentlichkeit wahrgenommen würden.

Diesen Befund unterstützen die quantitativen Ergebnisse der Studie von Alexandra Shajek, Katia Tödt, Annette Stelter, Phillip Gross und Nicolas Winterhager. Auf Basis einer Analyse von Publikationsdaten verzeichnen die Autor*innen im Zeitraum von 1995 bis 2019, und insbesondere ab 2005, stetig wachsende Zahlen an Fachpublikationen, die sich (laut Schlagwortsuche) mit digitaler Hochschulbildung beschäftigen. Überraschend für die Autor*innen ist dabei, dass sich zwischen den Fächern insgesamt deutlich weniger Unterschiede zeigen als von ihnen erwartet. Vielmehr lassen sich zwischen den Fächergruppen punktuelle 
Unterschiede im wissenschaftlichen Diskurs über die digitale Hochschulbildung hinsichtlich der Themenschwerpunkte (MOOCs, kollaboratives Lernen, Gamification) beobachten.

Dass die Gestaltung der Digitalisierung innerhalb wie außerhalb der Hochschulen auch ethische Herausforderungen zu bewältigen hat, verdeutlicht schließlich der Beitrag von Claudia Lemke, Dagmar Monett, Gert Faustmann und Kathrin Kirchner. Die Autor*innen stellen in ihrem Beitrag einen Ansatz zur Begriffsbestimmung digitaler Ethik und Anwendungsmöglichkeiten im Bildungsbereich vor. Das entwickelte Rahmenwerk setzt universelle ethische Grundsätze in Relation zu den handelnden Akteur*innen und wendet diese in Form einer narrativen Ethik beispielhaft an, um die Prinzipien praktisch zu vermitteln.

\subsection{Kooperationen und Netzwerke}

Teil zwei reflektiert drei politische Ebenen, die für Kooperationen und die Arbeit in Netzwerken maßgeblich sind. Der Beitrag von Tina Ladwig und Christiane Arndt zu Landeshochschulverbünden adressiert die regionale Zusammenarbeit im Rahmen von formalisierten Hochschulverbünden innerhalb der Grenzen einzelner Bundesländer. Die Autorinnen liefern eine fundierte Übersicht über Landesinitiativen in der Bundesrepublik und deren Motive, Ziele und Umsetzungsansätze. Zudem werden kumulierte Einschätzungen von Expert*innen zu einem potenziellen Mehrwert von bundesländerübergreifenden Kooperationen vorgestellt.

Hier knüpft der Beitrag von Astrid Book, Harald Gilch und Klaus Wannemacher mit einer bundesweiten Perspektive an. Auf Basis einer Sekundärauswertung von Umfragedaten, die 2018 für eine Studie zur Digitalisierung von Hochschulen in Deutschland erhoben wurden, untersuchen die Autor*innen, welche Rahmenbedingungen für Kooperationen besonders förderlich sind und welche Spezifika Hochschulen aufweisen, die systematisch auf Kooperationen in den Bereichen Lehre, Forschung und Verwaltung setzen. Aus den Ergebnissen der Sekundäranalyse leiten sich einmal mehr die Empfehlungen für Hochschulleitungen ab, in IT-Governance zu investieren und Digitalisierung in Hochschulstrategien zu verankern.

Florian Rampelt, Dominic Orr, Alexander Knoth und Renata Suter werfen schließlich einen Blick auf den Europäischen Hochschulraum. Der Beitrag arbeitet die Bedeutung der Digitalisierung für den Bologna-Prozess und die aus diesem entstandene European Higher Education Area (EHEA) heraus. Dabei identifizieren die Autor*innen zentrale Aktionsfelder für die Gestaltung der digitalen 
Transformation in der Hochschulbildung auf europäischer Ebene und diskutieren den Status quo in drei ausgewählten Schwerpunktbereichen mit Blick auf mögliche Zukunftsszenarien. Als Kernelemente der europäischen Zusammenarbeit werden die Bereiche Mobilität und Austausch, Kompetenzvermittlung sowie Qualitätssicherung vertieft betrachtet.

\subsection{Governance und Change Management}

Der dritte Teil widmet sich umfassenderen Fragen der Governance und des Changemanagements. Gleich drei Beiträge setzen sich mit dem zentralen Thema der Entwicklung von Hochschulstrategien auseinander.

Jannica Budde, die selbst die Organisation und Durchführung der Peer-toPeer-Strategieberatungen des Hochschulforums Digitalisierung mitverantwortet, entwickelt in ihrem Beitrag ein Modell mit sechs Grundmustern für strategische Narrative, mit denen Hochschulen Digitalisierungsprojekte begründen und diese in konkrete Bahnen lenken können. Eine zentrale Empfehlung von Budde ist, dass Hochschulen sich nicht allein auf Digitalisierung konzentrieren, sondern in einem möglichst umfassenden Verständigungsprozess ein individuelles Leitbild entwickeln sollten, an dem sich die Definition der strategischen Ziele und Optionen orientiert.

Auf Basis einer mindestens ebenso reichhaltigen Empirie entwickeln Marcel Graf-Schlattmann, Birte Thomsen, Melanie Wilde, Dorothee Meister und Gudrun Oevel ihr Modell der Kollektiven Veränderung, das Gelingensbedingungen für digitale Transformationsprozesse an Hochschulen identifiziert und systematisiert. Zwei Phänomene solcher Veränderungen werden in dem Beitrag näher diskutiert: die Projektförmigkeit als zentrales Organisationsmuster der digitalen Transformation an Hochschulen und das Verhältnis von Dynamik und Synchronisation, das aus Sicht der Autor*innen für die Nachhaltigkeit von Digitalisierungsprojekten erfolgskritisch ist.

Der Beitrag von Joachim Metzner erweitert die Diskussion um eine dritte Perspektive, indem er auf inhaltliche Defizite in der bisherigen Strategiebildung verweist. Diese führt Metzner auf das Fehlen einer politischen Dimension in hochschulischen Strategien einerseits und ein unklares Bildungsverständnis seitens der Politik andererseits zurück. Metzner formuliert vor diesem Hintergrund jeweils vier Empfehlungen für die Gestalter*innen in Hochschulen und Politik.

Vor dem Hintergrund der Strategiediskussion verweist der Beitrag von Falk Scheidig und Monika Holmeier auf spezifischere Herausforderungen, die sich quasi als Folge von Digitalisierungsbestrebungen an Hochschulen - aus einem 
zunehmenden Einsatz von digitalen Technologien in Studium und Lehre und der daraus resultierenden Datafizierung ergeben. Neuen Chancen einer datengetriebenen Optimierung von Lernumgebungen und -prozessen, die unter dem Stichwort Learning Analytics diskutiert werden, stehen anspruchsvolle Implementierungsprozesse sowie datenschutzrechtliche und ethische Bedenken gegenüber. Scheidig und Holmeier schlagen deshalb einen Orientierungsrahmen für Hochschulen vor, der aus institutioneller Perspektive 25 Handlungsanforderungen für einen sensiblen, potenzialorientierten und organisationsbewussten Umgang mit lehr- und lern-bezogenen Daten skizziert.

Dass die Gestaltung der Lehr- und Lernbedingungen an Hochschulen nicht nur von oben erfolgen sollte, verdeutlichen die folgenden zwei Beiträge. Frederic Denker, Ronny Röwert und Alexa Böckel plädieren für neue Formen der Partizipation von Studierenden in der Hochschulentwicklung, um die Bedürfnisse der Lernenden auch in strategischen Entscheidungen angemessen zu berücksichtigen. Empirisch speist sich der Beitrag aus den Erfahrungen der studentischen HFD-Arbeitsgruppe und Zukunfts-AG DigitalChangeMaker. Denker, Röwert und Böckel liefern ein leitliniengestütztes Phasenmodell der Studierendenbeteiligung und übersetzen dieses in Handlungsempfehlungen für Studierende und Hochschulverantwortliche.

Die Studie von Christina Gloerfeld untersucht didaktische Veränderungen durch Digitalisierung an der FernUniversität in Hagen und fokussiert dabei auf Partizipation an Lehr-/Lernprozessen. Die Studie erfasst Lehr-/Lernprozesse systematisch mithilfe eines analytischen Rahmenmodells, das aus sechs allgemeindidaktischen Modellen abgeleitet ist. Das Fazit der Autorin fällt eher verhalten aus: Die Realisierung der Potenziale für neue Handlungsräume und -praxen in Lehre und Lernen, die auch bestehende Hierarchien zwischen Lehrenden und Lernenden aufbrechen könnten, steckt noch in einem Anfangsstadium. Grundlegende Strukturen seien bislang wenig transformiert worden. Gloerfelds Ergebnisse liefern aber Impulse und Reflexionsansätze für den Umgang mit überzogenen Erwartungshaltungen in Bezug auf Veränderungen im Lehren und Lernen.

Die beiden Beiträge zu Partizipation komplementieren die vorangehenden Untersuchungen zu Strategiebildung und Change Management. Neben der Partizipation an Veränderungsprozessen ist die Frage der Inklusion respektive Inklusivität ein weiterer Schlüsselaspekt, den es im Zuge der digitalen Transformation der Hochschullehre und -governance zu berücksichtigen gilt. Isabel Zorn stellt in ihrem Beitrag pointiert die rechtlichen Vorgaben, organisatorischen Herausforderungen und Potenziale einer barrierearmen inklusiven „Hochschule für alle“ dar. Für die Gestaltung der Digitalisierung in Studium und Lehre schlägt die Autorin 
eine Orientierung an den Grundsätzen des Universal Designs vor, um die Vision und Chancen einer diskriminierungsfreien Hochschule zu verwirklichen.

Ein weiteres Themenfeld, das grundlegend für die Weiterentwicklung des Lehrens und Lernens an Hochschulen ist und damit in strategischen Überlegungen Berücksichtigung finden sollte, ist die Gestaltung zukünftiger - physischer und hybrider - Lernräume. Zwei Beiträge befassen sich deshalb mit Lernarchitekturen.

Katja Ninnemann führt in das Konzept von „Onlife Learning Spaces“ ein und verdeutlicht, dass ein ganzheitliches Verständnis des Lernraums Hochschule weit über Fragen der (Neu-)Gestaltung des (physischen) Lernraums Campus hinausgeht. Die Erkenntnisse ihrer Forschung implizieren einen grundlegenden Perspektivwechsel der Lernraumgestaltung, in dem die Entwicklung hybrider Lernumgebungen priorisiert werden sollte.

Richard Stang, Christine Gläser, Anke Petschenka und Alexandra Becker verstärken dieses Plädoyer für hybride Lehr- und Lernraumkonstellationen und eine entsprechende Strategieentwicklung ebenso wie den Appell der DigitalChangeMaker, die Perspektive der Studierenden zu berïcksichtigen. Auf Basis der Forschungsergebnisse des Projektes „Lernwelt Hochschule“ diskutieren die Autor*innen besonders relevante Aspekte für die Gestaltung der Zukunft im Modus der Digitalisierung.

Die letzten beiden Beiträge des dritten Buchteils beschäftigen sich mit neuen Formen der Anerkennung und Anrechnung von Prüfungsleistungen und weisen damit ebenfalls eine strategische Dimension auf. Hans Pongratz und Matthias Gottlieb liefern eine Hilfestellung für die Bewertung und Strategieentwicklung von digitalen Bildungsnachweisen (Digital Credentials). Diese wird explorativ anhand von definierten Zielen, der Analyse gegenwärtiger Fragestellungen und diskutierten Erfolgsfaktoren im Zusammenhang mit der Einführung und Nutzung von Digital Credentials an Hochschulen entwickelt. Die Ergebnisse von Pongratz und Gottlieb deuten in darauf hin, dass die Wirtschaftsinformatik ein zentrales Bindeglied für weitere Forschung und Entwicklung in diesem Bereich sein kann.

Anastasia Bertini, Annelie Pentenrieder, Jan Rebentisch, Samer Schaat und Robert Rentzsch diskutieren schließlich geteilte Herausforderungen und Erwartungen, die von verschiedenen Akteur*innen an die Entwicklung von DigitalCredentials-Systemen gestellt werden. Die empirische Basis dafür sind vier internationale Hochschulkooperationen mit deutscher Beteiligung, die die BlockchainTechnologie für die automatische Verifizierung der Nachweise nutzen. Der Beitrag vermittelt dabei auch die zugrunde liegenden Technologien, die perspektivisch für manipulationssichere Nachweise benötigt werden. 


\subsection{Kompetenzen und Curricula für das digitale Zeitalter}

Teil vier des vorliegenden Sammelbands adressiert Schlüsselkompetenzen und die Vermittlung von (digitalen) Kompetenzen im digitalen Zeitalter. Die ersten beiden Beiträge geben Einblicke in die Bedeutung und Vermittlung von Future Skills in der Hochschulbildung.

Ulf-Daniel Ehlers präsentiert mit dem „Future-Skills-Triple-Helix-Modell der Handlungsfähigkeit in emergenten Praxiskontexten" eine sowohl empirisch als auch bildungstheoretisch fundierte Konzeption des Gegenstands (und liefert damit auch eine gute Einführung in die Diskussion), die auf multimethodischen, mehrteiligen Studien fußt. Das Modell identifiziert 17 Skill-Profile in drei Dimensionen, darunter Fähigkeiten wie Selbst- und Ambiguitätskompetenz sowie ethische Kompetenz.

Der zweite Beitrag von Marija Stambolieva untersucht die Beziehung zwischen Arbeitsmarkt und Hochschulbildung sowie den Wandel der Berufsprofile von Ingenieurwissenschaftler*innen und damit verbundenen Anforderungen an Hochschulabsolvent*innen am Beispiel der Automobilindustrie. Die Autorin adressiert das Spannungsfeld zwischen veränderten Bedürfnissen in Unternehmen und hochschulpolitisch abgestimmten Kompetenzprofilen, auf denen die Gestaltung der Curricula in Hochschulen basieren. Stambolieva plädiert dabei für eine gleichwertige Berücksichtigung der Beschäftigungsbefähigung (Employability) bei der Curriculumentwicklung und einen Dialog zwischen Hochschule und Wirtschaft.

In einer zunehmend digitalisierten Welt lässt sich Data Literacy als Schlüsselkompetenz begreifen, die bislang jedoch noch nicht flächendeckend in der Hochschulbildung gefördert wird. Ausgehend von diesem Befund diskutiert der Beitrag von Matthias Bandtel, Leonie Kauz und Natalia Weißker Herangehensweisen an die Definition von Lernzielen, Möglichkeiten der curricularen Integration und geeignete didaktische Ausgestaltungen von Lehr-Lern-Settings sowie potenzielle Umsetzungsstrategien. Die Autor*innen wollen damit Impulse und Handlungsempfehlungen für Data Literacy Education für Studierende aller Fächer geben.

Antje Michel und Tobias Seidl vertiefen die Diskussion zur Curriculumentwicklung durch eine allgemeinere Auseinandersetzung mit drei zentralen Herausforderungen: den Stellenwert der Digitalisierung als kompetenzorientierter Lerninhalt, die Flexibilisierung von Curricula innerhalb von Akkreditierungszyklen zwecks Integration von digitalkompetenzorientierten Lernzielen und Lehr-Lern-Formen sowie die digitale Kompetenzentwicklung der Lehrenden. Der Beitrag liefert einen fachlichen Überblick sowie Impulse für die (Weiter-)Entwicklung von Studiengängen. 
Der Erwerb und die Fähigkeit zur Vermittlung von Kompetenzen sind in der Ausbildung von Lehrer*innen in einem doppelten Sinne von Bedeutung, weil angehende Lehrkräfte diese Kompetenzen an Schulen weitervermitteln sollen. Die Lehrer*innenbildung hat damit eine breitere gesellschaftliche Tragweite. Die letzten drei Beiträge in diesem Buchteil beschäftigen sich deshalb mit diesem Thema.

Sue-Ann Bäsler untersucht die Implikationen der Medienbildung in der Ausbildung von Lehrkräften mit einem qualitativen Ansatz. Für die Analyse der Bedeutung medienbezogener Vorstellungen von Lehramtsstudierenden nutzt Bäsler das Konzept des medialen Habitus und entwickelt es weiter. Aus den Ergebnissen der Studie leitet die Autorin Vorschläge für eine Verbesserung der Medienbildung sowie der medienpädagogischen Ausbildung von Lehrkräften ab. Hier lautet ein Vorschlag (einmal mehr), die Studierenden an der Ausgestaltung digitaler Lehre und Medienbildung zu beteiligen. Bäsler konstatiert in ihrem Fazit aber auch, dass die Entwicklung der Medienbildung in der deutschen Lehrkräftebildung grundsätzlich auf einem guten Weg sei.

Quasi komplementär zu Bäsler analysieren Charlott Rubach und Rebecca Lazarides die digitalen Kompetenzselbsteinschätzungen Studierender in der Lehrkräftebildung mit quantitativen Methoden. Auf Basis der Umfrageergebnisse lassen sich drei unterschiedliche Typen von Lehramtsstudierenden identifizieren, die sich signifikant in Bezug auf ihren Studienabschluss, ihre Einstellungen zur Nutzung digitaler Medien und ihr Nutzungsverhalten unterscheiden. Auch diese Ergebnisse haben praktische Implikationen für die Gestaltung der Hochschul- und Lehrkräftebildung, die die Autorinnen am Ende ihres Beitrags aufzeigen.

Swantje Borukhovich-Weis, Inga Gryl, Ewa Laczkowska und Björn Bulizek präsentieren schließlich die Ergebnisse einer qualitativen Begleitstudie zu einer Inverted-Classroom-Veranstaltung in der Lehrer*innenprofessionalisierung, die um Methoden der Selbststeuerung und Gamification angereichert wurde. Die Studie liefert weitere Erkenntnisse zur Gestaltung digitaler Lehr-Lern-Räume und markiert insbesondere Aspekte der Lernumgebung, die von den befragten Studierenden als lernfördernd oder lernhemmend erachtet wurden. So sind die Ergebnisse auch in Verbindung mit den oben genannten Beiträgen zu Lernarchitekturen sowie den Gamification-Beiträgen im folgenden letzten Teil des Sammelbands aufschlussreich. 


\subsection{Didaktik}

Der fünfte Teil des HFD-Sammelbands befasst sich mit den Möglichkeiten, Hochschullehre im digitalen Zeitalter interessant, interaktiv und auf den Lernerfolg fokussiert zu gestalten. Die in diesem Teil versammelten Beiträge zeugen nicht zuletzt vom Innovationsgeist und Engagement der Lehrenden und Mitarbeiter*innen in Didaktik- oder Supporteinrichtungen, die die Lehre unter den veränderten Bedingungen der Digitalisierung nahezu täglich unmittelbar und in der Interaktion mit Studierenden gestalten. In diesem Zusammenhang zeigen die Beiträge, dass es über gewisse technische Bedingungen und Kompetenzen hinaus innovativer didaktischer Konzepte bedarf. Dafür eignen sich diverse Ansätze, die aus anderen Bereichen stammen, wie zum Beispiel der Welt der analogen und digitalen Spiele (Gamification), aus agilen Methoden des Projekt- und Innovationsmanagements, Konzepten zur Förderung nachhaltiger Entwicklung bis hin zu den Features, die zeitgemäße Online-Plattformen bieten. In den Beiträgen wird die Zentrierung auf die Lernendenperspektive (Shift from Teaching to Learning) ebenso deutlich wie die Bestrebungen, digitale Lehre nicht in Form einer Kopie der Präsenzlehre umzusetzen, sondern die ihr innewohnenden Logiken und damit ihr eigenständiges Potenzial auszuschöpfen.

Im Beitrag von Thomas Bröker, Thomas Voit und Benjamin Zinger werden Erkenntnisse aus der analogen Spielewelt für Innovationen in der digitalen Didaktik eruiert. Spiele und Spielmuster sowie die darin enthaltenen Motivationsfaktoren lassen sich den Autoren zufolge für die Konzeption digitaler Lehr-Lern-Formate nutzen. Auf Basis einer qualitativen und quantitativen Auswertung von 30.000 Spielen des Deutschen Spielearchivs sowie der Reflexion digitaler Spielwelten entwickeln Bröker, Voit und Zinger eine systematische Vorgehensweise, die eine „spielerische Haltung“ fördern soll. In dem Beitrag wird auch die praktische Anwendung dieses Baukastens am Beispiel einer Vorlesung illustriert.

Daniel Tolks und Michael Sailer skizzieren den empirischen Stand der Forschung zu Gamification sowie einschlägige theoretische Modelle der Wirkung von Gamification auf Lernerfolg und Motivation. Der Beitrag betrachtet zudem einzelne Spielelemente wie Punkte, Leaderboards, Badges, Performance Graphs und andere und prüft sie auf ihre didaktische Eignung. Neben allgemeineren Empfehlungen für den Einsatz von Gamification in der Hochschulbildung legen die Autoren die Wirkungsweisen einzelner Spielelemente dar und liefern damit 
eine konkrete Hilfestellung für alle, die über den Einsatz in der eigenen Lehre nachdenken. ${ }^{2}$

Sven Ivens und Konstantin Kaiser erörtern die Frage, inwiefern sich die digitale Lernumgebung eines Online-Planspiels dazu eignet, Hochschullehre in Deutschland digitaler, kooperativer und internationaler zu gestalten. Der Beitrag reflektiert die - wachsende - wissenschaftliche Diskussion über Online-Planspiele in der Hochschulbildung und leitet daraus Forschungsfragen ab. Diese Fragen dienen der Analyse eines Online-Planspielseminars, das erstmals im Wintersemester 2019/2020 mit Studierenden der Politikwissenschaft an den Universitäten Antwerpen, Göttingen und Krakau durchgeführt wurde. Das Beispiel dieses innovativen Lehr-Lern-Formats tangiert also auch das Feld der internationalen Kooperationen in der Hochschullehre und gibt darüber hinaus erste Empfehlungen zur Frage, wie sich Drop-out-Raten in arbeitsintensiven Online-Veranstaltungen reduzieren lassen.

Die Schnittstelle von Didaktik und Technik wird in besonderem Maße in dem Beitrag von Tobias R. Ortelt, Claudius Terkowsky, Andrea Schwandt, Marco Winzker, Anke Pfeiffer, Dieter Uckelmann, Anja Hawlitschek, Sebastian Zug, Karsten Henke, Dominik May und Johannes Nau adressiert. Remote-Labore ermöglichen Studierenden (ingenieurwissenschaftlicher Studiengänge) an einigen Hochschulen die ortsunabhängige Nutzung von Laboren, Maschinen und Robotern. Diese online bedienbaren Lehr-Lern-Räume eignen sich in vielfacher Hinsicht für den Erwerb von (Schlüssel-)Kompetenzen im digitalen Zeitalter. Die Autor*innen beschreiben anhand von fünf Fallbeispielen, ${ }^{3}$ wie die Umsetzung und Integration von Remote-Laboren in Studium und Lehre gelingen kann, verdeutlichen aber auch, welche Herausforderungen nach wie vor bestehen.

Um die Förderung des digitalen - in Verbindung mit fachlichem und personalem - Kompetenzerwerbs geht es auch in dem Beitrag von Nico Sturm und Heike Rundnagel. Explorativ untersuchen Sturm und Rundnagel den Einsatz der digital angereicherten agilen Lernmethode „eduScrum“ in der Hochschullehre. Dazu werten sie die Selbsteinschätzungen von Studierenden über den eigenen Kompetenzzuwachs unter Bezug auf drei einschlägige Referenzrahmen aus, ordnen die Ergebnisse ein und liefern damit stichhaltige Belege für die Eignung der Methode „,eduScrum: digital“ für Studierende aller Fächer.

\footnotetext{
${ }^{2}$ In dem Beitrag wird zudem die HFD Community Working Group „Netzwerk Serious Games und Gamification for Health“ vorgestellt, in der sich Daniel Tolks engagiert.

${ }^{3}$ Es handelt sich dabei um eine systematisierte Betrachtung von fünf Remote-Laboren der HFD Community Working Group (CWG) „Remote-Labore in Deutschland“ entlang der Kriterien Technik, Didaktik und Organisation. Die Autor*innen sind überwiegend Mitglieder der CWG.
} 
Auch der folgende Beitrag von Hans Jörg Schmidt greift eine agile Problemlösungsmethode auf, die sich zur Förderung von grundlegenden Kompetenzen wie Teamfähigkeit und interdisziplinärer Zusammenarbeit eignet. Der Autor gibt einen konzeptorientierten Überblick über den Einsatz von Design Thinking (DT) an Hochschulen und verortet die Methode im hochschuldidaktischen und DT-spezifischen Forschungsdiskurs. Zudem arbeitet er Einsatzfelder, Bezüge zu verwandten Methoden, Stärken, Herausforderungen und Forschungsfragen heraus und bezieht diese auf Anforderungen, die die digitale Transformation an die Hochschuldidaktik stellt. Dabei interpretiert Schmidt Design Thinking als „Prozesspromotor“, der die mit einem ,doppelten Technologiedefizit“ konfrontierte Hochschuldidaktik auf dem Weg zur digitalen Hochschulbildung voranbringen kann.

Digitales Lernen und Lehren zeichnen sich grundlegend durch zwei Entwicklungslinien aus: Skalierung und Individualisierung. Die letzten beiden Beiträge zeigen am Beispiel von digitalen Lernplattformen das besondere Potenzial der Öffnung von Bildungsangeboten. Wie vor allem der erste Beitrag deutlich macht, besteht eine Herausforderung darin, das Spannungsverhältnis von Skalierung und Individualisierung in der Gestaltung von Lernplattformen zu harmonisieren.

Dana-Kristin Mah und Julia Hense erörtern die Frage, welche vorrangig digitalen Lern-/Lehrformate und Lernsettings geeignet sein könnten, um Wissen und Kompetenzen im Bereich der Künstlichen Intelligenz (KI) zu vermitteln, und welche Anforderungen eine digitale Lehr-Lern-Plattform erfüllen muss. Dazu untersuchen die Autorinnen systematisch die Lern-/Lehrformate auf 15 etablierten digitalen Lernplattformen. Der Beitrag liefert damit auch eine Übersicht über den Markt der Plattformen. Die Analyseergebnisse werden abschließend mit Blick auf ihre Implikationen für die Gestaltung der Plattform „KI-Campus - Die Lernplattform für Künstliche Intelligenz" diskutiert.

Dass sich Plattformen, E-Learning und Gamification-Elemente auch eignen, um ein Bewusstsein für gesellschaftliche Herausforderungen wie den Klimawandel und die Grenzen unseres Wachstums zu fördern, macht der letzte Beitrag des Sammelbands deutlich. Florian Kohler und Alexander Siegmund zeigen, wie gerade die Gestaltung der Digitalisierung in Studium und Lehre genutzt werden kann, um das Konzept der „Bildung für nachhaltige Entwicklung“ (BNE) in der Hochschullandschaft zu verankern und darüber vermittelt einen gesellschaftlichen Beitrag zur Erreichung von Nachhaltigkeitszielen zu leisten. BNE selbst zielt darauf ab, Lernende zu befähigen, die Auswirkungen des eigenen Handelns auf die Welt zu verstehen und verantwortungsvolle, zukunftsgerichtete Entscheidungen treffen zu können. 


\section{Without further ado ...}

Wir freuen uns über diesen weiteren Meilenstein in der relativ jungen Geschichte des Hochschulforums Digitalisierung und bedanken uns nochmals herzlich bei allen Autor*innen für die sportliche Ausarbeitung ihrer Beiträge unter den Bedingungen des Jahres 2020. Allen Leser*innen wünschen wir eine gewinnbringende Lektüre des ersten HFD-Sammelbands. Wir laden Sie ein, den gesamten Band oder einzelne Beiträge mit allen Interessierten zu teilen und für ihre Zwecke unter Beachtung der CC-BY-4.0-Lizenzbestimmungen - zu nutzen. Schließlich hoffen wir, dass wir mit dieser Publikation auch einen kleinen Beitrag zur Gestaltung der Digitalisierung in Studium und Lehre leisten und dass die Beiträge einige Denkanstöße geben. Ihre Fragen, Anregungen, Kritik oder Ideen für neue Ansätze nehmen wir beim Hochschulforum Digitalisierung gerne entgegen. Lassen Sie sich von den folgenden 33 Beiträgen inspirieren.

\section{Literatur}

Hochschulforum Digitalisierung. (2016). The Digital Turn - Hochschulbildung im digitalen Zeitalter (Arbeitspapier, 27). Berlin: Hochschulforum Digitalisierung. https://doi.org/10. 5281/zenodo.4282305.

Open Access Dieses Kapitel wird unter der Creative Commons Namensnennung 4.0 International Lizenz (http://creativecommons.org/licenses/by/4.0/deed.de) veröffentlicht, welche die Nutzung, Vervielfältigung, Bearbeitung, Verbreitung und Wiedergabe in jeglichem Medium und Format erlaubt, sofern Sie den/die ursprünglichen Autor(en) und die Quelle ordnungsgemäß nennen, einen Link zur Creative Commons Lizenz beifügen und angeben, ob Änderungen vorgenommen wurden.

Die in diesem Kapitel enthaltenen Bilder und sonstiges Drittmaterial unterliegen ebenfalls der genannten Creative Commons Lizenz, sofern sich aus der Abbildungslegende nichts anderes ergibt. Sofern das betreffende Material nicht unter der genannten Creative Commons Lizenz steht und die betreffende Handlung nicht nach gesetzlichen Vorschriften erlaubt ist, ist für die oben aufgeführten Weiterverwendungen des Materials die Einwilligung des jeweiligen Rechteinhabers einzuholen.

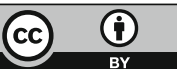

